\title{
MIOMATOSIS UTERINA E INFERTILIDAD: INDICACIONES DE TRAMIENTO CONVENCIONAL
}

\author{
CONVENTIONAL TREATMENT FOR UTERINE \\ MYOMATOSIS-INDUICED INIFERTILITIY \\ Jaime Saavedra, M.D.* \\ Recibido: mayo 9/2002 - Revisado: julio 22/2002 - Aceptado: noviembre 20/2002
}

\section{RESUMEN}

Los fibromas son relativamente comunes en las pacientes en edad reproductiva y son exclusivamente responsables de infertilidad y abortos en una pequeña $(5 \%)$ pero significante proporción de pacientes. Aproximadamente el $50 \%$ de las mujeres con infertilidad y miomas uterinos se embarazan después de miomectomía. Una proporción relativamente alta de mujeres con historia de abortos recurrentes se embaraza después miomectomía. Lo más importante, hay una disminución significativa en la tasa de abortos en el primer y segundo trimestre. La evidencia sugiere que la mayoría de mujeres que desean embarazarse son capaces de hacerlo en el primer año, con una caída de estas tasas en forma aguda después de este tiempo. Esto se puede atribuir a la recurrencia de los fibromas. Por tanto, la cirugía se debería realizar cuando la mujer este lista para iniciar una fa-

\footnotetext{
Profesor Titular. Departamento de Ginecología y Obstetricia. Universidad del Valle, Cali - Colombia. Jefe del Servicio de Ginecología Hospital Universitario del Valle - Cali, Colombia. Director del Programa de Entrenamiento en Cirugía Endoscópica Ginecológica - Clínica Los Andes, Cali -Colombia. Director General del Centro de Medicina Reproductiva, Fecundar, Cali- Colombia. Presidente de la Sociedad Colombiana de Cirugía Endoscópica.
}

milia. El sitio, número y tamaño de los miomas así como la experiencia del cirujano junto con la preferencia de la paciente puede influenciar la opción del manejo.

Son indicaciones para realizar cirugía en una mujer que esté considerando la posibilidad de embarazo por los métodos naturales o por reproducción asistida la presencia de un mioma submucoso o un mioma intramural que distorsione la cavidad uterina, fibromas mayores de 5 centímetros y múltiples fibromas. Para fibromas intramurales menores de 5 centímetros y fibromas subserosos la historia reproductiva debe ser una consideración importante en la determinación en lo tocante a la necesidad de una intervención quirúrgica.

Palabras claves: leiomioma, miomectomía, infertilidad, reproducción asistida

\section{SUMMARY}

Fibroids are a fairly frequent occurrence in the reproductive age group and are exclusively responsible for both infertility and pregnancy wastage in a small (5\%) but significant proportion 
of patients. Approximately 50\% of women with infertility and uterine myomas conceive after myomectomy. A slightly higher proportion of women with history of recurrent pregnancy loss conceive following myomectomy. More importantly, there is highly significant reduction in early and mid trimester miscarriage rates. Evidence suggests that most women wish to conceive are able to do so in the first year, with pregnancy rates dropping sharply after this time. This may be attributed to recurrence of fibroids. If possible, therefore, the surgery should be timed to take place when the woman is ready to start a family. The site, number and size of the myomas as well as the expertise of the surgeon along with patient preference may all influence the management option. A submucous fibroid or an intramural fibroid distorting the uterine cavity, fibroids $>5 \mathrm{~cm}$ and multiple fibroids are all indications for surgery in woman considering a pregnancy. For relatively small $(<5 \mathrm{~cm})$ intramural or subserosal fibroids, reproductive history is an important consideration in counseling the patient regarding the need for surgical intervention.

Key words: leiomyoma, myomectomy, infertility, assisted reproduction

\section{INTRODUCCIÓN}

Los leiomiomas uterinos (fibromas) son los tumores más comunes encontrados en la mujer. Su ocurrencia se incrementa con la edad; y se encuentran presentes entre un 20\% a 50\% de las mujeres mayores de 30 años de edad. ${ }^{1,2}$

Las manifestaciones clínicas de estos tumores benignos son variables y la mayoría de ellos son asintomáticos. La sintomatología y su severidad dependen del tamaño, posición, y número de ellos presentes. $^{1}$

La influencia de los fibromas uterinos en reproducción se manifiesta en varias vías. Los fibromas uterinos, especialmente los de tipo submucoso, están asociados con un aumento de riesgo de aborto espontáneo, parto pretérmino, presentaciones anormales durante el trabajo de parto, obstrucción del canal cervical, hemorragia posparto, sepsis puerperal. ${ }^{3,4}$ Sin embargo, el efecto de su presencia en la fertilidad es aun objeto de controversia. ${ }^{5}$

Estos tumores están presentes tanto en mujeres fértiles como en infértiles. Sin embargo, un número significativo (43\%) de mujeres embarazadas con fibromas tienen historia de infertilidad antes del embarazo. ${ }^{6}$

Esta revisión se enfocará en el papel de estos tumores comunes en el comportamiento reproductivo de la mujer y la justificación de realizar miomectomía en el momento que la mujer desee fertilidad.

\section{PATOGÉNESIS}

Los fibromas son tumores benignos compuestos de fibras musculares lisas. Townsen y cols. en $1970,{ }^{6}$ demostró usando la isoezima 6-glucosa fosfato deshidrogenasa, que cada una de las células dentro del leiomioma se origina de una sola célula miometrial. Estudios citogenéticos también sugieren que la transformación y crecimiento neoplásico de un mioma es el resultado de rearreglos cromosómicos en células del miometrio. ${ }^{7}$ Las diferentes tasas de crecimiento son el resultado de diferentes anormalidades citogenéticas dentro de cada leiomioma. La mayoría de estos tumores tienen un cariotipo normal, mientras múltiples miomas dentro del mismo útero no tienen relación citogenética y por tanto cada uno se origina independientemente. ${ }^{8}$

\section{ETIOLOGÍA}

Aunque los fibromas son una patología común, su etiología es pobremente comprendida. Hay una gran variedad de factores que pueden jugar un papel sinérgico en el crecimiento de estos neoplas- 
mas benignos, entre los más evidentes de ellos están los estrógenos.

Los leiomiomas no son comunes antes de la menarca, crecen durante el periodo reproductivo especialmente durante el embarazo y regresan después de la menopausia, lo cual da una evidencia clínica de que los estrógenos juega un papel fundamental en el crecimiento de éstos. Se ha encontrado una alta concentración de receptores para estrógenos en el tejido miomatoso que circunda el miometrio durante todo el ciclo menstrual..$^{9,10}$

El metabolismo de los estrógenos dentro de los miomas esta alterado, tal es que el estradiol $\left(E_{2}\right)$ es pobremente convertido a estrona, ${ }^{11}$ haciendo el medio ambiente del mismo mioma relativamente estrogénico a pesar de que niveles hormonales sistémicos son normales en la mujer con mioma uterino. ${ }^{12}$

En cuanto al papel de la progesterona en el desarrollo de los miomas es contradictorio. Goldzieher y cols., ${ }^{13}$ encontraron evidencia histológica de cambios degenerativos en leiomiomas después de la administración de altas dosis de progesterona (medrogestrona $25 \mathrm{mg}$ por 21 días), no encontrando efectos benéficos después de la administración de dosis pequeñas (2 mg de noretindrona de 1 a 30 meses).

Kawaguchi y cols., ${ }^{14}$ encontraron grandes cambios mitóticos en leiomiomas removidos quirúrgicamente durante la fase secretora, sugiriendo que la progesterona puede disparar el crecimiento de estos tumores (esto se puede explicar por el hecho de que los estrógenos aumentan la expresión de los receptores de progesterona).

Finalmente, Murphy y cols., ${ }^{15}$ encontraron que la administración de la antiprogesterona mifepristone (RU486) por 3 meses resulta en una disminución del volumen de los leiomiomas hasta en un $87 \%$. Hay evidencia que la hormona de crecimiento (HC) actúa sinérgicamente con el estradiol induciendo el crecimiento de los leiomiomas. ${ }^{16}$
Sharara y cols. ${ }^{16}$ reportaron la presencia de receptores de hormona de crecimiento (HC) en estos tumores. Cohen y cols. ${ }^{17}$ observaron una alta incidencia de leiomiomas (81\%) en pacientes con acromegalia, en quienes el exceso de la hormona de crecimiento se piensa tenga un papel importante como causa de miomas.

Reportes recientes han descrito la presencia de receptores para el factor 1 de crecimiento similar a la insulina (IGF-1). ${ }^{16,18,19}$ Este y otros factores como son IGF-II ${ }^{7}$ y el factor crecimiento epidermoide $^{20}$ promueven el crecimiento de los leiomiomas antes que iniciar su crecimiento.

\section{CLASIFICACIÓN \\ DE LOS LEIOMIOMAS}

Los diferentes leiomiomas pueden afectar el resultado reproductivo en diferente extensión, y los resultados de las intervenciones quirúrgicas para cada tipo pueden variar. Los leiomiomas se pueden clasificar en submucosos, intramurales, o subserosos. ${ }^{21}$ Infortunadamente no hay consenso en la literatura acerca de cómo definir estas categorías.

La clasificación sugerida para los leiomiomas de acuerdo a su localización en el útero es la siguiente:

1. Submucoso. Un leiomioma submucoso es aquel que distorsiona la cavidad uterina. La Sociedad Europea de Histeroscopia clasifica los leiomiomas submucosos. ${ }^{22}$ En tres subtipos: tipo 0 - leiomioma pediculado sin extensión intramural, tipo I - sésil con extensión intramural del leiomioma $<50 \%$ y tipo II sésil con extensión intramural del 50\% o más. El grado de extensión intramural se puede evaluar por ultrasonido, o por histeroscopia observando el ángulo entre el leiomioma y el endometrio unido a la pared uterina.

2. Intramural (intersticial). Un leiomioma que no distorsiona la cavidad uterina y menos de un $50 \%$ de él sobresale en la superficie serosa del útero. 
3. Subseroso. Un leiomioma es considerado subseroso si más del 50\% del leiomioma sobresale de la superficie serosa del útero. El leiomioma subseroso puede ser sésil o pedunculado.

\section{LEIOMIOMAS E INFERTILIDAD}

El papel de los leiomiomas como causa posible de infertilidad ha sido objeto de un debate considerable. Mientras muchas mujeres se embarazan fácilmente, otras tienen problema para ello. La localización anatómica de los leiomiomas es altamente relevante en cuanto a la posibilidad de producir infertilidad; en orden decreciente sería: submucoso, intramural y subseroso.

Varios mecanismos se han propuesto para explicar los posibles efectos adversos de los leiomiomas en infertilidad:

1. Distorsión de la cavidad endometrial.

2. Aumento de la distancia en el viaje de esperma para alcanzar las trompas uterinas.

3. Obstrucción del ostio tubárico

4. Alteración en trasporte de los gametos

5. El aumento del tamaño y contorno del útero puede alterar la implantación

6. Alteración en el suministro sanguíneo al endometrio, afectando así la nidación

7. Interferencia con la función de la unión uterotubarica, comprometiendo flujo sanguíneo, o causando irritabilidad miometrial.

Los leiomiomas, según The American Fertility Society Guideline for Practice, ${ }^{23}$ pueden estar asociados con un $5 \%$ a $10 \%$ de casos de infertilidad, pero los leiomiomas como factor único solamente están presentes en un 2\% a 3\% de los casos de infertilidad. $^{3}$

En términos generales, el papel de los miomas en infertilidad se ha evaluado indirec- tamente por los resultados de fertilidad después de miomectomía.

En una revisión realizada por Buttran y Reiter ${ }^{3}$ ellos describieron que de 76 pacientes sometidos a miomectomía abdominal sin ninguna causa aparente de infertilidad el 54\% se embarazó después de cirugía, tasas similares fueron reportadas por Verkauf ${ }^{2}$ quien describió 79 pacientes similares de los cuales $59,5 \%$ se embarazaron pos miomectomía, otros estudios recientes confirman estos hallazgos ${ }^{24-27}$ sugiriendo un mejoramiento en las tasas de embarazo después de miomectomía.

En un metanálisis reciente, ${ }^{28}$ se reporto una tasa de embarazo en tres estudios en los que se realizo miomectomía como causa única de infertilidad de miomas intramurales y subserosos del 58\% al 65\%, estas tasas bajan a un 38\% cuando se encuentran asociadas otras causas de infertilidad.

\section{LEIOMIOMAS Y RESULTADO REPRODUCTIVO}

Se ha observado que aproximadamente de 1\% a 4\% de los embarazos están asociados con leiomiomas. ${ }^{29-31}$ En general, el 80\% de leiomiomas uterinos presentes durante el embarazo permanecen del mismo tamaño, o se vuelven más pequeños durante el curso del embarazo. ${ }^{30}$

Los leiomiomas pueden producir complicaciones diversas durante el embarazo. La primera de ellas son los abortos. La tasa de abortos espontáneos es alta si la implantación ocurre sobre un mioma submucoso. ${ }^{32}$ Los leiomiomas próximos a la placenta están asociados a sangrados tempranos y abortos espontáneos.

La localización de leiomioma con relación a la placenta parece ser más importante que su tamaño. ${ }^{29,33}$ Los miomas intramurales o subserosos $<3$ $\mathrm{cm}$ son considerados a menudo de poca significación clínica. ${ }^{30-33}$ 
No obstante no hay datos prospectivos que sugieran de sí o no los leiomiomas intra murales o subserosos aumentan la tasa de abortos, y en que extensión ellos son causa abortos recurrentes. En un estudio retrospectivo T.C.Li ${ }^{26}$ mostró que la tasa de perdidas de embarazo después de miomectomía fue del 24\% siendo esta significativamente mas baja que antes de miomectomía 60\%, en este estudio se sugiere que los miomas son causa de perdidas en el orden de $40 \%$ y del $17 \%$ en el primero y segundo trimestre, reduciéndose dramáticamente después de miomectomía.

Estos resultados sugieren que la miomectomía se debería considerar en aquellas mujeres con historia de abortos, especialmente en el segundo trimestre o de dos pérdidas en el primer trimestre. Cuando ha habido únicamente una pérdida en el primer trimestre, los pro y contra de la cirugía se deben discutir cuidadosamente con la paciente y en tratamiento se deben tener en cuenta otros factores como la edad, infertilidad, y otros síntomas ginecológicos como menorragia. A las mujeres con pérdidas recurrentes en el primer trimestre, se les deben realizar una investigación exhaustiva para excluir otras causas posibles diferentes a los leiomiomas.
Aparte de los abortos, los miomas pueden producir otras complicaciones durante el embarazo: parto prematuro, dolor abdominal debido a degeneración roja, desprendimiento de placenta, retardo del crecimiento intrauterino, obstrucción del trabajo de parto y hemorragia posparto. El riesgo de todas las complicaciones se ha reportado tan alto como del $77 \%{ }^{34}$

\section{IMPACTO DE LOS LEIOMIOMAS EN CONCEPCIÓN ASISTIDA}

Hay cinco estudios retrospectivos que en conjunto examinan el impacto de los miomas en los resultados en concepción asistida ${ }^{35-39}$. Los resultados para cada tipo de mioma son analizados en forma separada en la tabla 1 (A, B, C, D).

La tasa de embarazo por embrión trasferido en miomas submucoso, intramural y subseroso fueron de 9\%, 16\% y 37\% respectivamente, comparado con un $30 \%$ en los casos controles. Los resultados son consistentes con la observación de que los miomas submucosos son los que más efecto detrimental hacen sobre las tasas de embarazo, los miomas intra murales tienen un impacto modesto, los miomas subserosos tienen poco impacto o ningún impacto en las tasas de embarazo.

Tabla 1A. Resultado de concepción asistida como tratamiento en mujeres con miomas

Pacientes con miomas submucosos

\begin{tabular}{|c|c|c|c|c|c|c|c|c|}
\hline \multirow{2}{*}{$\begin{array}{l}\text { Autor } \\
\text { Farthi } 1995\end{array}$} & \multirow{2}{*}{$\begin{array}{c}\text { Casos } \\
18 \\
\end{array}$} & \multirow{2}{*}{$\begin{array}{c}\begin{array}{c}\text { Embriones } \\
\text { transferidos }\end{array} \\
55\end{array}$} & \multicolumn{2}{|c|}{$\begin{array}{c}\text { Tasa de } \\
\text { embarazo }\end{array}$} & \multicolumn{2}{|c|}{$\begin{array}{c}\text { Tasa de } \\
\text { nacimiento }\end{array}$} & \multicolumn{2}{|c|}{$\begin{array}{l}\text { Tasa de } \\
\text { aborto }\end{array}$} \\
\hline & & & $5 / 55$ & $28 \%$ & $3 / 5$ & $60 \%$ & $2 / 5$ & $40 \%$ \\
\hline Elgar Geva 1998 & 9 & 10 & $1 / 10$ & $10 \%$ & NS & & NS & \\
\hline TOTAL & 27 & 65 & $6 / 65$ & $9,2 \%$ & $3 / 5$ & $60 \%$ & $2 / 5$ & $40 \%$ \\
\hline \multicolumn{9}{|c|}{ Pacientes controles } \\
\hline Autor & Casos & $\begin{array}{c}\text { Embriones } \\
\text { transferidos }\end{array}$ & \multicolumn{2}{|c|}{$\begin{array}{c}\text { Tasa de } \\
\text { embarazo }\end{array}$} & \multicolumn{2}{|c|}{$\begin{array}{c}\text { Tasa de } \\
\text { nacimiento }\end{array}$} & \multicolumn{2}{|c|}{$\begin{array}{l}\text { Tasa de } \\
\text { aborto }\end{array}$} \\
\hline Farthi 1995 & 50 & 127 & $32 / 127$ & $25 \%$ & $24 / 32$ & $75 \%$ & $8 / 32$ & $25 \%$ \\
\hline Elgar Geva 1998 & 249 & 318 & $98 / 318$ & $30 \%$ & $78 / 98$ & $80 \%$ & $20 / 98$ & $20 \%$ \\
\hline TOTAL & 299 & 445 & $130 / 445$ & $29,2 \%$ & $102 / 130$ & $78,5 \%$ & $28 / 130$ & $22,5 \%$ \\
\hline
\end{tabular}


Tabla 1B. Resultado de concepción asistida como tratamiento en mujeres con miomas

Pacientes con miomas intramurales

\begin{tabular}{lcccccccc}
\hline Autor & Casos & $\begin{array}{c}\text { Embriones } \\
\text { transferidos }\end{array}$ & \multicolumn{2}{c}{$\begin{array}{c}\text { Tasa de } \\
\text { embarazo }\end{array}$} & \multicolumn{2}{c}{$\begin{array}{c}\text { Tasa de } \\
\text { nacimiento }\end{array}$} & \multicolumn{2}{c}{$\begin{array}{c}\text { Tasa de } \\
\text { aborto }\end{array}$} \\
\hline Elgar Geva 1998 & 46 & 55 & $9 / 55$ & $\mathbf{1 6 \%}$ & $6 / 9$ & $\mathbf{6 7 \%}$ & $3 / 9$ & $\mathbf{3 3 \%}$ \\
\hline Pacientes controles & 249 & 318 & $98 / 318$ & $\mathbf{3 0 \%}$ & $78 / 98$ & $\mathbf{8 0 \%}$ & $20 / 98$ & $\mathbf{2 0 \%}$ \\
\hline
\end{tabular}

Tabla 1C. Resultado de concepción asistida como tratamiento en mujeres con miomas

Pacientes con miomas subserosos

\begin{tabular}{|c|c|c|c|c|c|c|c|c|}
\hline \multirow{2}{*}{$\begin{array}{l}\text { Autor } \\
\text { Scoud } 1992 \\
\end{array}$} & \multirow{2}{*}{$\begin{array}{c}\text { Casos } \\
11 \\
\end{array}$} & \multirow{2}{*}{$\begin{array}{c}\begin{array}{c}\text { Embriones } \\
\text { transferidos }\end{array} \\
24\end{array}$} & \multicolumn{2}{|c|}{$\begin{array}{c}\text { Tasa de } \\
\text { embarazo }\end{array}$} & \multicolumn{2}{|c|}{$\begin{array}{c}\text { Tasa de } \\
\text { nacimiento }\end{array}$} & \multicolumn{2}{|c|}{$\begin{array}{l}\text { Tasa de } \\
\text { aborto }\end{array}$} \\
\hline & & & $10 / 24$ & $42 \%$ & $5 / 10$ & $50 \%$ & $5 / 10$ & $50 \%$ \\
\hline Elgar Geva 1998 & 33 & 41 & $14 / 41$ & $34 \%$ & $11 / 14$ & $79 \%$ & $3 / 14$ & $21 \%$ \\
\hline TOTAL & 44 & 65 & $24 / 65$ & $37 \%$ & $16 / 24$ & $67 \%$ & $8 / 24$ & $33 \%$ \\
\hline \multicolumn{9}{|c|}{ Pacientes controles } \\
\hline Autor & Casos & $\begin{array}{c}\text { Embriones } \\
\text { transferidos }\end{array}$ & \multicolumn{2}{|c|}{$\begin{array}{c}\text { Tasa de } \\
\text { embarazo }\end{array}$} & \multicolumn{2}{|c|}{$\begin{array}{c}\text { Tasa de } \\
\text { nacimiento }\end{array}$} & \multicolumn{2}{|c|}{$\begin{array}{l}\text { Tasa de } \\
\text { aborto }\end{array}$} \\
\hline Scoud 1992 & 1357 & 2018 & $586 / 2018$ & $29 \%$ & $484 / 586$ & $83 \%$ & $102 / 586$ & $17 \%$ \\
\hline Elgar Geva 1998 & 249 & 318 & $98 / 318$ & $30 \%$ & $78 / 98$ & $80 \%$ & $20 / 98$ & $20 \%$ \\
\hline TOTAL & 1606 & 2336 & $684 / 2336$ & $29 \%$ & $562 / 684$ & $82 \%$ & $122 / 684$ & $18 \%$ \\
\hline
\end{tabular}

Tabla 1D. Resultado de concepción asistida como tratamiento en mujeres con miomas

Pacientes con miomas subserosos / intramurales

\begin{tabular}{lcccccccc}
\hline Autor & Casos & $\begin{array}{c}\text { Embriones } \\
\text { transferidos }\end{array}$ & \multicolumn{2}{c}{$\begin{array}{c}\text { Tasa de } \\
\text { embarazo }\end{array}$} & \multicolumn{2}{c}{$\begin{array}{c}\text { Tasa de } \\
\text { nacimiento }\end{array}$} & $\begin{array}{c}\text { Tasa de } \\
\text { aborto }\end{array}$ \\
\hline Ramzy 1998 & 39 & 39 & $15 / 39$ & $39 \%$ & $9 / 15$ & $60 \%$ & $6 / 15$ & $40 \%$ \\
\hline Stovall 1998 & 91 & 91 & $34 / 91$ & $37 \%$ & $30 / 34$ & $88 \%$ & $4 / 34$ & $12 \%$ \\
\hline Farhi 1995 & 28 & 86 & $25 / 86$ & $29 \%$ & $15 / 25$ & $60 \%$ & $10 / 25$ & $40 \%$ \\
\hline TOTAL & 158 & 216 & $74 / 216$ & $34 \%$ & $54 / 74$ & $73 \%$ & $20 / 74$ & $27 \%$ \\
\hline
\end{tabular}

Pacientes controles

\begin{tabular}{lcccccccc}
\hline Autor & Casos & $\begin{array}{c}\text { Embriones } \\
\text { transferidos }\end{array}$ & \multicolumn{2}{c}{$\begin{array}{c}\text { Tasa de } \\
\text { embarazo }\end{array}$} & \multicolumn{2}{c}{$\begin{array}{c}\text { Tasa de } \\
\text { nacimiento }\end{array}$} & \multicolumn{2}{c}{$\begin{array}{c}\text { Tasa de } \\
\text { aborto }\end{array}$} \\
\hline Ramzy 1998 & 367 & 367 & $123 / 367$ & $34 \%$ & $104 / 123$ & $85 \%$ & $19 / 123$ & $15 \%$ \\
\hline Stovall 1998 & 91 & 91 & $48 / 91$ & $53 \%$ & $44 / 48$ & $92 \%$ & $4 / 91$ & $8 \%$ \\
\hline Farhi 1995 & 50 & 127 & $32 / 127$ & $25 \%$ & $24 / 32$ & $75 \%$ & $8 / 32$ & $25 \%$ \\
\hline TOTAL & 508 & 585 & $203 / 585$ & $35 \%$ & $172 / 203$ & $85 \%$ & $31 / 203$ & $15 \%$ \\
\hline
\end{tabular}


Las tasas de aborto en los diferentes tipos de miomas fueron: submucosos, 40\%, intramural, 33\% y subserosos, 21\% comparado con un total 153/ $930(16,5 \%)$ entre todos los pacientes controles de los cinco estudios. Mientras todo parece mostrar, nuevamente, la tendencia similar a las tasas embarazo, es un descenso en orden de importancia: miomas submucosos, miomas intra murales y subserosos, no obstante él número de casos es muy pequeño del total para hacer una conclusión firme.

Todos los estudios discutidos han sido retrospectivos, con un número pequeño de pacientes y se ha hecho poca distinción entre miomas intramurales y subserosos.

Hart y cols. ${ }^{40}$ realizaron el primer gran estudio prospectivo controlado del efecto de los miomas intramurales (de $5 \mathrm{~cm}$ o menos) en el resultado de la concepción asistida, en la tabla 2 se presentan los datos demográficos de este. La tasa por embrión trasferido de embarazo, implantación y embarazo en curso son significantemente mas bajas en el grupo de pacientes con miomas, $23,3 \%, 11,9 \%$ y $15,1 \%$ respectivamente comparado con $34,1 \%, 20,2 \%$ y $28,3 \%$ en el grupo control (tabla 3).

Los autores concluyen que los pacientes con pequeños miomas intramurales a quienes se sometieron a reproducción asistida presentan una tasa de embarazo en curso significantemente reducida cuando se compara con los pacientes sin miomas intramurales.

De la información disponible hasta el momento se concluye que paciente con miomas submucosos o intra murales estos se les deben retirar antes de FIV, mientras que aquellos con miomas subserosos pueden estar tranquilos que es improbable de que estos tengan un efecto adverso en la tasa de implantación y de nacimientos. Los miomas mayores de $5 \mathrm{~cm}$ se deben considerar individualmente, teniendo en cuenta la historia reproductiva, por ejemplo número de abortos. ${ }^{26}$

Factores que afectan la concepción y los resultados reproductivos después de miomectomía

\section{EDAD DE LA PACIENTE}

La edad se ha encontrado que tiene un impacto significativo en la tasa de embarazo, con tasas bajas de concepción espontáneamente o en reproducción asistida cuando la mujer es mayor de 35 años. ${ }^{26,38}$ Es aceptado que las mujeres mayores tienen menos probabilidad de concebir que las mujeres jóvenes. ${ }^{41-42}$

\section{HISTORIA DE INFERTILIDAD}

Acien y Quereda ${ }^{41}$ encontraron que una historia de infertilidad mayor de 3 años de duración,

Tabla 2. Estudio prospectivo controlado para determinar el efecto de los miomas intramurales en el resultado de reproducción asistida

\section{Datos demográficos}

\begin{tabular}{lcccc}
\hline Parámetros & $\begin{array}{c}\text { Pacientes } \\
\text { Controles } \mathbf{n = 3 2 2}\end{array}$ & $\begin{array}{c}\text { Pacientes con } \\
\text { Miomas n=112 }\end{array}$ & Diferencia & $\boldsymbol{P}$ \\
Edad al inicio del tratamiento & $34,6 \pm 3,9$ & $36,4 \pm 3,9$ & 19 & $<0,0001$ \\
\hline Años de infertilidad & $3,2 \pm 3,7$ & $36,6 \pm 3,5$ & 0,4 & 0,38 \\
\hline $\begin{array}{l}\text { Número de ciclos previos } \\
\text { al tto de FIV/ICSI }\end{array}$ & $0,7 \pm 1,1$ & $0,6 \pm 1,2$ & $-0,1$ & 0,26 \\
\hline FSH basal pre tratamiento & $7,9 \pm 10,8$ & $4,6 \pm 10,7$ & $-0,3$ & 0,84 \\
\hline
\end{tabular}

Hart y cols.: Human Reproduction 2001;16:2411-2417. 


\begin{tabular}{|c|c|c|c|c|}
\hline & Pacientes & \multirow{2}{*}{$\begin{array}{c}\text { Pacientes con } \\
\text { Miomas } n=112\end{array}$} & \multirow[t]{2}{*}{ Diferencia } & \multirow[t]{2}{*}{$p$} \\
\hline & Controles $n=322$ & & & \\
\hline $\begin{array}{l}\text { Número de folículos el día } \\
\text { de la aspiración }\end{array}$ & $11,7+6,0$ & $12,1+6,6$ & 0,4 & NS \\
\hline Número de oocitos aspirados & $9,3+5,5$ & $10,1+6,3$ & 0,8 & NS \\
\hline $\begin{array}{l}\text { Número de embriones aptos } \\
\text { para transferir }\end{array}$ & $4,4+3,8$ & $4,5+4,4$ & 0,1 & NS \\
\hline Número de embriones transferidos & $2,1+0,6$ & $2,0+0,8$ & $-0,1$ & NS \\
\hline Tasa de implantación & $20,2 \quad(16,8-24,2)$ & $11,9 \quad(7,7-18,2)$ & $-8,3$ & 0,018 \\
\hline Tasa de embarazo & $34,1 \quad(28,7-39,9)$ & $23,3 \quad(14,8-33,6)$ & $-10,8$ & 0,018 \\
\hline Embarazo en curso & $28,3 \quad(23,1-33,8)$ & $15,1 \quad(8,3-24,4)$ & $-13,2$ & 0,018 \\
\hline
\end{tabular}

Hart y Col: Human reproduction 2001;16:2411-2417

afecta negativamente las tasas de concepción. Igualmente, la tasa de embarazo se reduce hasta en un $20 \%$ en presencia de factores adicionales de infertilidad. ${ }^{29}$

Mujeres sin historia de infertilidad tienen una probabilidad mayor de concebir después de miomectomía que aquellas con historia de infertilidad. ${ }^{27}$ La miomectomía debería ser considerada en pacientes con historia de infertilidad inexplicada mayor de 2 años de duración..$^{27,43}$

\section{ABORTO}

Los miomas uterinos están asociados con un aumento en la tasa de aborto en el primero y segundo trimestres de embarazo; esta tasa se reduce significativamente después de miomectomía. ${ }^{26,28}$

\section{TAMAÑO DE LOS LEIOMIOMAS}

En las primeras revisiones del tema, se sugirió que un tamaño uterino mayor de 10 semanas antes de la cirugía estaba asociada con un pobre resultado reproductivo después de la miomectomía ${ }^{3}$. Sudik y cols. ${ }^{44}$ encontraron que la tasa de embarazo después de miomectomía de miomas con un volumen mayor de $100 \mathrm{~mL}$, aproximadamente 8 cm de diámetro fue significativamente más alta que en esas de pequeños miomas.

Ellos sugieren que los miomas grandes interfieren con mayor frecuencia la fertilidad que los pequeños, por tanto la remoción los miomas grandes resulta en el restablecimiento de esta. En una revisión de 27 estudios, Vercellini y cols. ${ }^{28}$ no pudo encontrar una evidencia conclusiva, en cualquier vía, si el número, tamaño y localización de los miomas antes de la miomectomía influenciaba las tasas posoperatorias. El tamaño del útero no fue considerado como un factor determinante en el resultado reproductivo en otros dos estudios ${ }^{27,46}$.

\section{NÚIMERO DE LEIOMIOMAS}

La tasa de embarazo fue significativamente baja en mujeres cuando se les removieron más de cinco fibromas. ${ }^{44}$ Esta reducción puede ser atribuida al aumento del número de incisiones y subsiguiente formación de adherencias.

En un estudio de cincuenta pacientes que deseaban embarazo, Berkeley y cols. ${ }^{42}$ encontraron que a mujeres a las cuales se les había removido múltiples miomas presentaban dificultad en concebir en el posquirúrgico. Por otro lado, otros 
autores observaron que no había ninguna diferencia en la tasa de embarazo después de miomectomía independientemente del tamaño y número de fibromas removidos 26,27 .

\section{LOCALIZACIÓN}

\section{DE LOS LEIOMIOMAS}

La localización de los leiomiomas no parece afectar la tasa de embarazo después de la cirugía, sea o no su localización submucosa y prescindiendo de si o no la cavidad uterina fue penetrada $27,42,44$.

\section{TIEMPO DE CONCEPCIÓN POST-CIRUGÍA}

La mayoría de las mujeres que conciben lo hacen en el primer año después de miomectomía. ${ }^{27,42,44}$ Esta consideración es importante cuando el médico tiene que decidir si opera una mujer asintomática, quien no está considerando un embarazo en el futuro inmediato.

\section{MIOMECTOMÍA POR LAPAROTOMÍA}

La miomectomía por laparotomía debe ser la ruta de elección cuando se tiene un mioma subseroso o intramural mayor de 7 centímetros, cuando se tiene que remover múltiples miomas mayores de 5 centímetros y cuando se supone que se va entrar a la cavidad uterina. En el procedimiento quirúrgico se debe aplicar los principios de la técnica microquirúrgica para evitar la formación de adherencias que generen posteriormente un factor peritoneal de infertilidad.

La tasa de embarazo reportada después de miomectomía en varios estudios retrospectivos (tabla 4), está entre 50\% y 68\%, con una tasa de aborto espontáneo del 6\% al 40\%, y una tasa de nacimientos del 57\% al 93\%, calculada como porcentaje del número total de embarazos.
La operación cesárea fue el modo de nacimiento en cerca del $50 \%$ de las mujeres después de miomectomía (86/177).

En dos amplias revisiones realizadas por Verkauf $^{2}$ y más recientemente por Vercellini, ${ }^{28}$ estos sugieren que más del 50\% de las pacientes con infertilidad o abortos espontáneos conciben después de miomectomía. El corto intervalo entre la cirugía y la concepción sugiere que la miomectomía tiene un importante valor en el tratamiento de pacientes con fibromas sin ninguna otra causa de infertilidad inexplicada ${ }^{2}$.

\section{MIOMECTOMÍA POR HISTEROSCOPIA}

Neuwirth y Amin (1976) fueron los primeros en sugerir que la miomectomía histeroscópica era el tratamiento de elección de los miomas submucosos. Los miomas submucosos son responsables del $5 \%$ de todos los fibromas ${ }^{46}$, aunque en grupos seleccionados puede ser tan alto como $18 \% .{ }^{47}$

Comparada con la miomectomía abierta, la miomectomía histeroscópica está asociada a una menor incidencia de operación cesárea, ya que no hay escisión de la pared uterina. Se evitan la formación de adherencias pélvicas, las cuales son de ocurrencia común durante la miomectomía abierta. Los resultados de siete estudios retrospectivos de miomectomía histeroscópica se resumen en la tabla 5. Los resultados totales incluyendo, las tasas de embarazo (55\%) y tasas de nacimientos (80\%) son muy similares a aquellos obtenidos de miomectomía por laparotomía. Se encontró una tasa significantemente alta de embarazo y de nacimientos cuando se resecaron fibromas mayores de 2 centímetros comparados con mujeres a quienes se les resecaron fibromas menores de 2 centímetros mujeres con cavidad uterina normal.

Además, se observó que el tiempo para alcanzar la concepción después de la cirugía fue mucho 
Tabla 4. Resultado de miomectomía por laparotomía en pacientes infértiles

\begin{tabular}{lccccc} 
Autor & Casos & \multicolumn{2}{c}{ Tasa de embarazos } & \multicolumn{2}{c}{ Niños en casa } \\
\hline Berkeley 1983 & 50 & $25 / 50$ & $50 \%$ & $20 / 25$ & $80 \%$ \\
\hline Garcia \& Tureck 1984 & 15 & $8 / 15$ & $53 \%$ & $7 / 8$ & $88 \%$ \\
\hline Rosenfield 1986 & 23 & $15 / 23$ & $65 \%$ & $13 / 15$ & $57 \%$ \\
\hline Smith \& Uhlir 1986 & 32 & $16 / 32$ & $50 \%$ & $12 / 16$ & $75 \%$ \\
\hline Verkauf 1992 & 26 & $16 / 26$ & $50 \%$ & $10 / 16$ & $63 \%$ \\
\hline Gehlbach 1993 & 37 & $19 / 37$ & $51 \%$ & $14 / 19$ & $65 \%$ \\
\hline Sudik 1996 & 67 & $39 / 67$ & $58 \%$ & $31 / 51$ & $61 \%$ \\
\hline Acien \& Quereda 1996 & 40 & $27 / 40$ & $68 \%$ & $25 / 27$ & $93 \%$ \\
\hline Li et al 1999 & 51 & $29 / 51$ & $57 \%$ & $25 / 33$ & $76 \%$ \\
\hline Vercellini 1999a & 174 & $85 / 174$ & $49 \%$ & $92 / 104$ & $88 \%$ \\
\hline TOTAL & $\mathbf{4 6 5}$ & $\mathbf{2 7 9 / 4 6 5}$ & $\mathbf{6 0 \%}$ & $\mathbf{2 4 9 / 3 1 4}$ & $\mathbf{7 9 \%}$ \\
\hline
\end{tabular}

\begin{tabular}{lcccccc}
\multicolumn{2}{c}{ Tabla 5. Tasas de embarazo despues de miomectomia por } \\
histeroscopia en mujeres infertiles \\
Autor & Casos & Tamaño & Tasa de embarazos & \multicolumn{2}{c}{ Nacimientos } \\
\hline Corson \& Brooks 1991 & 13 & av $<4$ & $10 / 13$ & $77 \%$ & $8 / 11$ & $73 \%$ \\
\hline Valle, 1990 & 16 & av 3,2 & $10 / 16$ & $63 \%$ & $8 / 16$ & $80 \%$ \\
\hline Goldenberg et al 1995 & 15 & $3-6$ & $7 / 15$ & $47 \%$ & $6 / 7$ & $86 \%$ \\
\hline Hallez 1995 & 32 & $1-6,5$ & $18 / 32$ & $56 \%$ & $21 / 23$ & $91 \%$ \\
\hline Ciatras et al, 1999 & 41 & av $<4$ & $25 / 41$ & $61 \%$ & $23 / 25$ & $92 \%$ \\
\hline Varasteh et al 1999 & 36 & $<4$ & $19 / 36$ & $53 \%$ & $13 / 19$ & $68 \%$ \\
& & $<2$ & $4 / 12$ & $33 \%$ & $3 / 4$ & $75 \%$ \\
& & $<2$ & $15 / 24$ & $63 \%$ & $10 / 15$ & $67 \%$ \\
\hline Vercellini et al, 1999 & b45 & $<3,5$ & $19 / 45$ & $42 \%$ & $14 / 21$ & $67 \%$ \\
\hline TOTAL & $\mathbf{1 9 8}$ & & $\mathbf{1 0 8 / 1 9 8}$ & $\mathbf{5 5 \%}$ & $\mathbf{9 3 / 1 1 6}$ & $\mathbf{8 0} \%$ \\
\hline
\end{tabular}

menor en mujeres con grandes fibromas. No se encontró diferencia en las tasas de aborto espontáneo después de tratamiento en esos dos grupos.

Vercellini y cols. ${ }^{28}$ notaron una tasa alta de embarazo (10/22) cuando se resecaron fibromas submucosos pediculados (tipo I). Los fibromas grandes toman un mayor tiempo para resecarlos, requieren de un cirujano muy experimentado y frecuentemente se requiere de más de una intervención quirúrgica para removerlos completamente. En su estudio, únicamente una de seis mujeres con fibromas submucosos tipo II se embarazaron. ${ }^{28}$
Estos autores sugieren que es preferible una miomectomía abierta a una resección histeroscópica cuando se tiene que remover un fibroma submucoso con predominio intramural.

La miomectomía histeroscópica es procedimiento seguro, efectivo y relativamente simple para restaurar la fertilidad. No obstante fibromas submucosos tipo II (mayores de 5 centímetros) puede no ser conveniente manejarlos quirúrgicamente por histeroscopia; si se considera la cirugía histeroscópica en esta situación, es esencial tener la mano control 
laparoscópico y un cirujano histeroscopista muy experimentado.

\section{MIOMECTOMÍA}

\section{LAPAROSCÓPICA}

En el momento actual es posible realizar miomectomía laparoscópica de miomas subserosos o intramurales. Nezhat y Wallach han sugerido que el manejo laparoscópico es apropiado para miomas pedunculados o subserosos, pero también anotaron que la remoción de miomas pedunculados subserosos asintomáticos puede no estar justificado, ya que ellos no necesariamente pueden ser causa de infertilidad o aborto recurrente. ${ }^{48-49}$

El cierre adecuado del miometrio es indispensable para evitar el riesgo de ruptura uterina en un futuro embarazo ${ }^{43}$. La miomectomía laparoscópica de miomas intramurales requiere de un cirujano laparoscopista muy experimentado, si esto no es posible lo mejor es hacer la remoción de éstos por laparotomía.

Una técnica intermedia es realizar la remoción de los miomas por laparoscopia, y posteriormente hacer la extracción de estos mediante una micro laparotomía, y luego hacer el cierre de la incisión del miometrio utilizando la técnica microquirúrgica para evitar posteriormente la formación de adherencias post-cirugía.
El resultado reproductivo después de la miomectomía laparoscópica ha sido examinado en seis estudios (tabla 6). Las tasas de embarazo y nacimiento (47\% y 76\% respectivamente) son comparables a aquellas obtenidas después miomectomía abierta o por histeroscopia. Una vez nuevamente, la mayoría (más del 80\%) de las mujeres se embarazan durante el primer año después de la miomectomía.

\section{SELECCIÓN DEL PACIENTE Y RECOMENDACIONES}

Es importante realizar una evaluación cuidadosa de los riesgos y beneficios de la cirugía, antes de ofrecer cualquier procedimiento al paciente. Una valoración apropiada del paciente es indispensable. Se debe realizar un estudio de los problemas de infertilidad o de los abortos recurrentes antes de realizar cualquier cirugía. La historia clínica reproductiva detallada se debe complementar con estudios ecográficos, laparoscopia, histeroscopia y/ o histerosalpingograma. Esto ayudará a determinar el tamaño, número y localización de los miomas, con el fin de terminar la opción de manejo correcta, incluyendo la selección quirúrgica.

La mujer con infertilidad o una historia obstétrica pobre, a menudo consideran medidas temerarias que puedan mejorar su resultado reproductivo. Las mujeres deben ser asesoradas a fondo

Tabla 6. Tasas de embarazo después de miomectomía por laparoscopia

\begin{tabular}{lccccccc} 
Autor & Casos & \multicolumn{2}{c}{ Tasa embarazo } & Tasa de nacimiento & \multicolumn{2}{c}{ Tasa de cesáreas } \\
\hline Dubuisson et al 1996 & 21 & $7 / 21$ & $33,3 \%$ & $5 / 7$ & $71 \%$ & $4 / 5$ & $80 \%$ \\
\hline Miller et al 1996 & 40 & $30 / 40$ & $75 \%$ & $26 / 30$ & $87 \%$ & no declaró & \\
\hline Darai et al 1997 & 44 & $17 / 44$ & $39 \%$ & $11 / 19$ & $58 \%$ & $3 / 11$ & $27 \%$ \\
\hline Seinera et al 1997 & 34 & $5 / 34$ & $15 \%$ & $5 / 5$ & $100 \%$ & $5 / 5$ & $100 \%$ \\
\hline Ribeiro et al 1999 & 28 & $18 / 26$ & $64 \%$ & $14 / 18$ & $78 \%$ & $8 / 14$ & $57 \%$ \\
\hline Campo \& Garcea 1999 & 24 & $13 / 24$ & $54 \%$ & $11 / 13$ & $85 \%$ & $5 / 11$ & $45 \%$ \\
\hline TOTAL & $\mathbf{1 9 1}$ & $\mathbf{9 0 / 9 1}$ & $\mathbf{4 7 \%}$ & $\mathbf{6 8 / 9 0}$ & $\mathbf{7 6 \%}$ & $\mathbf{2 5 / 4 6}$ & $\mathbf{5 4 \%}$ \\
\hline
\end{tabular}


y estar completamente informadas de las diferentes opciones terapéuticas. El médico debe ser capaz de discutir las tasas de éxito esperadas, así como las posibles complicaciones.

\section{BIBLIOGRAFÍA}

1. Wallach EE. Myomectomy. En: Thompson JD, Rock JA, editors. TeLinde's operative gynecology. $7^{\text {th }}$ ed. Philadelphia: Lippincott, 1992; p. 647-662.

2. Verkauf BS. Myomectomy for fertility enhancement and preservation. Fertil Steril 1992; 58: 1-15.

3. Buttram VC, Reiter RC. Uterine leiomyomata: etiology, symptomatology and management. Fertil Steril 1981; 35: 433- 435.

4. Katz VL, Dotters DJ, Droegemueller W. Complications of uterine leiomyomas in pregnancy. Obstet Gynecol 1989; 73: 593-596.

5. Vollenhoven BJ, Lawrence AS, Healy DL. Uterine fibroids: a clinical review. Br J Obstet Gynaecol 1990; 97: 285- 298.

6. Towsend D, Sprks R, Baluda M. Unicellular histogenesis of uterine leiomyomas as determined by electrophoresis of glucose-6- phosphate dehydrogenase. Am J Obstet Gynecol 1970; 107: 11681172 .

7. Rein M, Friedman A, Stuart J, McLaughlin D. Fibroid and myometrial steroid receptors in women treated with gonadotropin realizing hormone against leuprolide acetate. Fertil Steril 1990; 53: 1018-1023.

8. Friedman A. Agonist therapy for uterine leiomyomata. En: Barbieri, R, Freidman A (eds.), Gonadropin releasing hormone analogs: applications in gynecology, Elsevier, New York 1991; p. 39-59.

9. Lumsden MA, West CP, Hawkins RA, Bramley TA, Rumgay L, Baird DT. The binding of steroids to myometrium and leimyomata in women treated with the gonadotropin releasing agonist, Zoladex (ICI 118630). J Endorinol 1989; 121:389-396.

10. SadanO, Van Iddekinge B, Van Gelderen CJ, Savage N, Becker PJ, Van Der Walt LA. Estrogen and progesterone receptor concentration in leiomyoma and normal myometrium. Ann Clin Biochem 1987; 24: 263-267.

11. Pollow K, Sinnecker G, Boquoi E, Pollow B. In vitro conversion of estradiol 17 beta into estrone in normal human myometrium and leiomyomas. J Clin Chem Clin Biochem 1978; 16: 493-502.

12. Maheax R, Lemay-Turcot l, Lemay A. Daily follicle stimulating hormone, leutinizing hormone, estradiol and progesterone in ten women harboring uterine leiomyomas. Fertil Steril 1986; 46: 205-208.

13. Goldzieher J, Maqueo M, Ricaud L, Aguilar J, Canales E. Induction of degenerative changes in uterine myomas by high dose progestin therapy. Am J Obstet Gynecol 1966; 96: 1078-1081.

14. Kawaguchi K, Fujii S, Konishi I. Mitotic activity in uterine leimyomas during the menstrual cycle. Am J Obstet Gynecol 1989; 160: 637-641.

15. Murphy A, Kettel L, Morales A. Regression of uterine leiomyomata in response to the antiprogesterone RU486. J Clin Endocrinol Metab 1993; 76: 513-517.

16. Sharara F, Neiman L. Growth hormone receptor messenger ribonucleotide acid expression in leiomyoma and surrounding myometrium. Am J Obstet Gynecol 1995; 178: 814-819.

17. Cohen O, Schindel B, Homburg R. Uterine leiomyomata a feature of acromegaly. Human Reprod 1998; 13: 1945-1946.

18. Guidice L, Irwing J, Dsupin B. Insulin like growth factor IGF, IGF binding proteins (IGF BP) and IGF receptor gene expression and IGFBP synthesis in human uterine leiomyomata. Human Report 1993; 89: 1796-1806.

19. Vollenhoven B, Herrington A, Healy D. Messenger ribonucleotide acid expression of the insulin like growth factors and their binding proteins in uterine fibroids and myometrium. J Clin Endocrinol Metab 1993; 76: 1106-1110.

20. Fayad Y, Tsibris J, Langenberg P, Robertson A. Human uterine leiomyomas cells, binding and growth responses to epidermal growth factor, plateletderived growth factor and insulin. Lab Invest 1989; 60: $30-35$. 
21. Tindall VR. Tumors of the corpus uteri. En: Jeffcate's Principles of Gynecology, 5 ${ }^{\text {th }}$ Ed, 1987; p. 417-444.

22. Wamsteker K, Emanuel M, Kruif JH. Transcervical hysteroscopic resection of submucous fibroids for abnormal uterine bleeding: results regarding the degree of intramural extension. Obstet Gynecol 1993; 82: 736-740.

23. American fertility Society. Guideline for Practice. Myomas and Reproductive Dysfunction. The American Fertility Society, Alabama, USA, 1992.

24. Garcia CR, Tureck RW. Submucosal leiomyomas and infertility. Fertil.Steril 1984; 42:16-19.

25. Gehlbach DL, Sousa RC, Carpenter SE, Rock JA. Abdominal myomectomy in the treatment of infertility. Int J Gynecol Obstet 1993; 40:45-50

26. Li TC, Mortimer R, Cooke ID. Myomectomy: a retrospective study to examine reproductive performance before and after surgery. Hum Reprod 1999; 14: $1735-1740$.

27. Rosenfeld DL. Abdominal myomectomy for otherwise unexplained infertility. Fertil Steril 1986; 46: 328-330.

28. Versellini P, Maddalena S, DeGiorgi O, Aimi G, Crosignani PG. Abdominal myomectomy for infertility: a comprehensive review. Hum Reprod 1998; 13:873-879.

29. Muram D, Gillieson M, Walters JH. Myomas of the uterus in pregnancy: Ultrasonographic follow up. Am J Obstet Gynecol 1980; 138: 16-19.

30. Rice JP, Kay HH, Mahony BS. The clinical significance of uterine leiomyomas in pregnancy. Am J Obstet Gyncol 1988; 160: 1212-1226.

31.Exacoustos C, Rosati P. Ultrasound diagnosis of uterine myomas and complications in pregnancy. Obstet Gynecol 1993; 82: 97-101.

32. Vollenhoven BJ, Lawrence AS, Healey DL. Uterine fibroids: a clinical review. Br J Obstet Gynaecol 1990; 97:285-298.

33. Rosati P, Bellati U, Exacoustos C, Angelozzi P, Mancuso S. Uterino myoma in pregnancy: utrasound study. Int J Gynecol Obstet 1989; 28: 109-117.
34. Phelan JP. Myomas and pregnancy. Obstet Gynecol Clin North America 1995; 22: 801-805.

35.Farhi J, Ashkenazi J, Feldberg D, Dicker D, Orvieto R, Ben Raphael Z. Effect of uterine leiomyomata on the results of in vitro fertilization treatment. Hum Reprod 1995; 10: 2576-2578.

36. Eldar-Geva T, Meagher S, Healy DL. Effect of intramural, subserosal, and submucuosal uterine fibroids on the outcome of assisted reproductive technology treatment. Fertil Steril 1998; 70: 687-691.

37. Seoud MA, Patterson R, Muasher SJ. Effects of myomas or prior myomectomy on in vitro fertilization (IVF) performance. J Assist Reprod Genet 1992; 9: 217-221.

38. Ramzy AM, Sattar M, Amin Y, Mansour RT, Serour GI, Aboulghar MA. Uterine myomata and outcome of assisted reproduction. Human Reprod 1998; 13: 198-202.

39. Stovall DW, Parrish SB, Van Voorhis, Hahn SJ, Sparks AET, SyropCH. Uterine leiomyomas reduce the efficacy of assisted reproduction cycles: results of a matched follow up study. Human Reprod 1998; 13: 192-197.

40. Hart R, Khalaf Y, Yeong Cheng Toh, Seed P, Taylor A, Braude P. A prospective controlled study of the effect of intramural uterine fibroids on the outcome of assisted conception. Hum Reprod 2001; 16: 2411 2417.

41. Acien P, Quereda F. Abdominal myomectomy: results of simple operative technique. Fertil Steril 1996; 65: 41-51.

42. Berkeley AS, DeCherney AH, Polan ML. Abdominal myomectomy and subsequent fertility. Surg Gynaecol Obstet 1983; 156: 319-322.

43. Dubuisson JB, Chapron C, Chavet X, Gregorakis SS. Fertility after laparoscopiic myomectomy of large intramural myomas: preliminary results. Hum Reprod 1996; 11: 518-522.

44. Sudik R, Husch K, Steller J, Daume E. Fertility and pregnancy outcome after myomectomy in sterility patients. Eur. J Obstet Gynecol Repro Biol 1996; 13: 192-197. 
45. Smith DC, Uhlir JK. Myomectomy as a reproductive procedure. Am J Obst Gynecol 1990; 162: 1476-1482.

46. Novak ER, Woodruff JD. Myoma and other benign tumor of the uterus In gynecologic and obstetric pathology; eighth edn, W.B Saunders, Philadelphia, $1979,260-278$.
47. Fedele L, Bianchi S, Dorta M, Brioschi D, Zanotti F, Versellini P. Trasvaginal ultrasonography versus hysteroscopy in the diagnosis of uterine submucous myomas 1991; 77: 745-748.

48. Nezhat C. The " cons" of laparoscopic myomectomy in women who may reproduce in the future. Int $J$ Fertil Menopausal Stud 1996; 41: 280-283.

49. Wallach EE, Vu KK. Myomata uteri and infertility. Obstet Gynecol Clin North America 1995; 22: 791-799. 